\title{
Effect of concentration on the formation time of diffraction rings in spatial self-phase modulation
}

\author{
Yilin He (何奕林), Jingdi Zhang (张景迪), Si Xiao (肖 思) ${ }^{*}$, Yingwei Wang (王迎威) ${ }^{* *}$, and Jun He (何 军) \\ Hunan Key Laboratory of Nanophotonics and Devices, School of Physics and Electronics, Central South University, Changsha 410083, China \\ *Corresponding author: sixiao@csu.edu.cn \\ ${ }^{* *}$ Corresponding author: wyw1988@csu.edu.cn \\ Received May 28, 2021 | Accepted July 16, 2021 | Posted Online September 15, 2021
}

\begin{abstract}
A new unsaturated wind-chime model is proposed for calculating the formation time of the diffraction rings induced by spatial self-phase modulation (SSPM) in molybdenum disulfide suspension. To optimize the traditional wind-chime model, the concentration variable of $2 \mathrm{D}$ materials was introduced. The results of the unsaturated wind-chime model match quite well with the SSPM experimental results of molybdenum disulfide. Based on this model, the shortest formation time of diffraction rings and their corresponding concentration and light intensity can be predicted using limited data. Theoretically, by increasing the viscosity coefficient of the solution, the response time of the diffraction ring, to reach the maximum value, can be significantly reduced. It has advanced significance in shortening the response time of photonic diodes.
\end{abstract}

Keywords: spatial self-phase modulation; wind-chime model; nonlinear optics.

DOI: 10.3788/COL202220.011901

\section{Introduction}

It has been 40 years since spatial self-phase modulation (SSPM) was discovered in $1981^{[1]}$. Since then, SSPM has developed rapidly due to the simple experimental device required to produce it, wide measurable wavelength range, strong diffracted beam, and feasibility for materials with a relatively large gap ${ }^{[2]}$. The research materials include a large number of two-dimensional (2D) materials, such as $\mathrm{Bi}_{2} \mathrm{TeSe}_{2}{ }^{[3]}$, graphene ${ }^{[4,5]}$, molybdenum disulfide ${ }^{[6-8]}$, MXenes ${ }^{[9-11]}$, and other ${ }^{[12-19]}$. Tremendous effort has been put into comprehending the underlying mechanism for the appearance of the SSPM patterns. The theoretical reports involve several factors such as concentration ${ }^{[20]}$, light intensity ${ }^{[21]}$, and thickness of a cuvette ${ }^{[22]}$. With the development of nonlinear optics ${ }^{[23-26]}$, today SSPM has become a ubiquitous, convenient, and efficient method for characterizing the thirdorder nonlinear optical properties of 2D materials ${ }^{[27,28]}$.

Recently, all-optical switching and information converters based on SSPM have been developed ${ }^{[29]}$. A nonreciprocal light propagation device has also been proposed based on the graphdiyne $/ \mathrm{SnS}_{2}$ structure $^{[30]}$. Furthermore, taking advantage of the strong light-matter interaction, a hybrid dimensional heterojunction-based logic gate is designed to realize the OR function with the optical method ${ }^{[31]}$. The successful preparation of graphdiyne-PMMA film ${ }^{[30]}$ and the Te@Bi quantum dots (QDs) nanotubes (NTs) PMMA film ${ }^{[31]}$ significantly promoted the progress of self-diffraction in photoelectric applications.
Nevertheless, photoelectric applications are inseparable from the key parameter of response time, but hardly ever reported.

The "wind-chime" model is an ideal model proposed by Zhao's group based on non-local electronic coherence theory, which can be used to calculate the formation time of the diffraction rings from the initial to maximum number of rings. When the light is incident on the sample, the nanosheets begin to be polarized. Initially, there is an arbitrary angle between the nanosheet and the electric field. Thanks to energy relaxation, the electric field reorients the nanosheets so that each domain contains an axis parallel to the polarization direction of the external field,

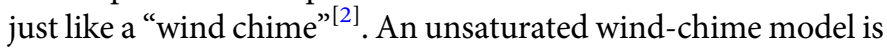
put forward in this work by introducing the concentration variable to satisfy different concentration conditions. These findings will no doubt promote the theoretical development of SSPM.

\section{Materials and Methods}

The $\mathrm{MoS}_{2}$ nanosheets were synthesized via conventional liquid phase exfoliation ${ }^{[32]}$. As a result, the size of the nanosheets is difficult to be uniformly perfect. Figure 1(a) shows the scanning electron microscope (SEM) image of $\mathrm{MoS}_{2}$. The nanosheets show an average size with hundreds of nanometers, which will induce typical SSPM diffraction rings. In previous reports, the results show that the size of the nanosheets will influence the 
nonlinear optical response when the size changes from hundreds of nanometers nanosheets to several nanometers QDs ${ }^{[20]}$. Here, the size changes within a smaller range than that of the previous report. So, we mainly explored the influence of the concentration on the formation time of the diffraction rings without considering the size dependent effect of SSPM. Scanning transmission electron microscopy (STEM) and energy-dispersive $\mathrm{X}$-ray spectroscopy (EDX) mapping images for $\mathrm{MoS}_{2}$ are presented in Fig. 1(b). The Mo and S elements show overlapped morphology structure, which confirmed the elemental composition of the as-prepared $\mathrm{MoS}_{2}$. The oxygen element can also be observed due to unavoidable oxidation during the preparation of the samples. An atomic force microscope (AFM) measurement was performed to characterize the thickness of the $\mathrm{MoS}_{2}$ nanosheets, and the results are shown in Figs. 1(c) and 1(d). The average thickness of $\mathrm{MoS}_{2}$ nanosheets is about $5.3 \mathrm{~nm}$.

The experimental setup of SSPM is shown in Fig. 2(a). A femtosecond (fs) laser regenerative amplifier from Spectra-Physics was utilized as the experimental light source. The laser is incident at a wavelength of $700 \mathrm{~nm}$ (it can be seen from Ref. [18] that at different wavelengths, the formation time of the diffraction rings is nearly the same) with a repetition frequency of $2 \mathrm{kHz}$ and a pulse width of about $40 \mathrm{fs}$. Due to the use of the fs laser, the heat generation is very limited during the nonlinear optical response measurement here. The focal length of the lens was $20 \mathrm{~cm}$, and the cuvette size was $1 \mathrm{~cm} \times 1 \mathrm{~cm} \times 4 \mathrm{~cm}$. While testing $\mathrm{MoS}_{2}$, its front surface was $10 \mathrm{~cm}$ away from the lens, and the back surface was $22.5 \mathrm{~cm}$ from the laser beam profiler (LBP2VIS2). The camera features a resolution array of $964 \times 724$, the acquisition mode is 12 bits, and the time resolution is up to 30 frames per second. (a)

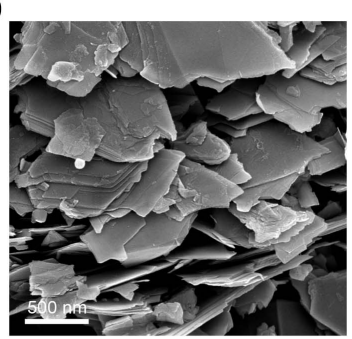

(c)

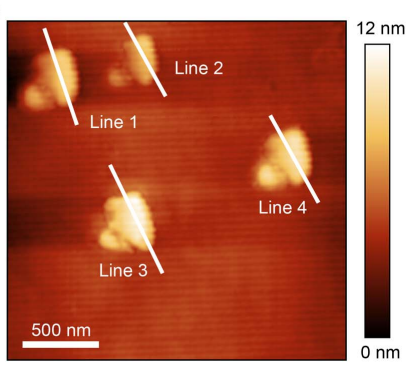

(b)

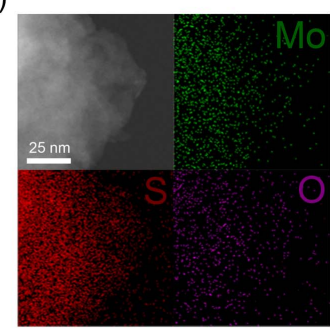

(d)

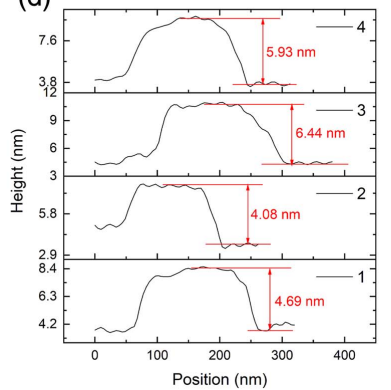

Fig. 1. (a) SEM image of $\mathrm{MoS}_{2}$ nanosheets. (b) STEM image and EDX mapping images of $\mathrm{MoS}_{2}$ nanosheets. (c) AFM image of $\mathrm{MoS}_{2}$. (d) The height profiles corresponding to the solid lines in (c).

\section{Results and Discussion}

When light passed through the sample, diffraction rings began to appear until the radius of the SSPM pattern reached its maximum [Figs. 2(b1)-2(b4)]. Then, the upper part of the diffraction rings collapsed downwards to form a stable conical structure that is vertically asymmetric [Fig. 2(b5)], which is caused by the thermal convection phenomenon ${ }^{[4]}$.

Furthermore, the reorientation of a 2D material can be explained according to the following process: under laser irradiation, photo-excited electrons and holes move in opposite directions, which polarizes the $2 \mathrm{D}$ material. The polarized $2 \mathrm{D}$ material would be reoriented parallel to the electric field to minimize the system's energy. The formation time of diffraction rings in Figs. 2(b1)-2(b4) is equal to the time required for the nanosheets reorientation in Figs. 2(c2)-2(c3). Moreover, the ring formation time $T$ can be calculated as follows ${ }^{[2]}$ :

$$
T=\frac{\varepsilon_{r} \pi \eta \xi r c}{1.72\left(\varepsilon_{r}-1\right) I h},
$$

where $r$ is the radius of the fragment, $h$ is the thickness, $\xi$ is a compensation value, $\varepsilon_{r}$ is the permittivity, $\eta$ is the solvent's viscosity, and $I$ is the laser intensity. However, the change in the sample concentration was not considered in this model. In this work, the improvement for different sample concentrations based on this model is proposed.

In the traditional model ${ }^{[2]}$, all nanosheets are considered to participate in the wind-chime model and contribute to the number of rings. Our previous work demonstrated that not all

(a)

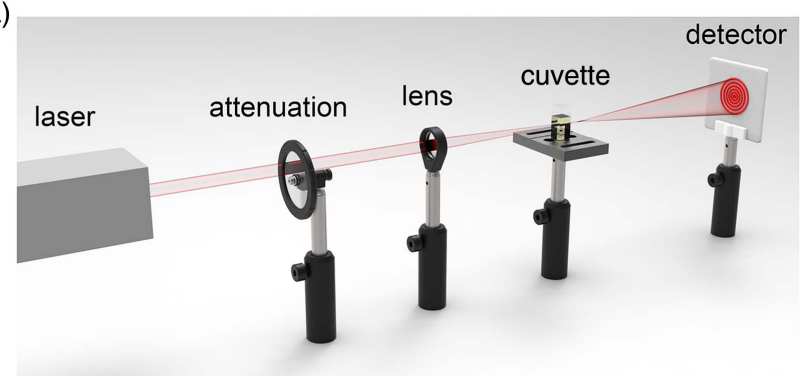

(b1)

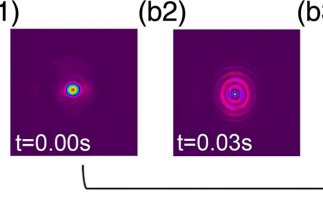

(b4)

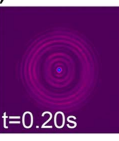

$(10)$

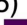

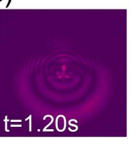

the formation time $T$

(c1)

(c2)

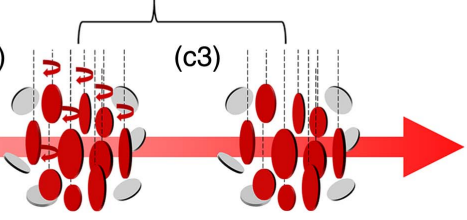

Fig. 2. (a) Schematic setup of SSPM. (b1)-(b5) The dynamic process of the diffraction ring patterns. (c1)-(c3) Unsaturated wind-chime model changes with the increase in time. 
fragments are involved in forming the wind-chime model ${ }^{[20]}$. In other words, the number of rings is related only to the number of nanosheets participating in the wind-chime model.

In fact, in the general "wind-chime" model, the total number of fragments has been determined once the sample solution is determined ${ }^{[20]}$ :

$$
A_{\text {total }}=\frac{\rho R^{2} l}{r^{2} h}
$$

where $R$ is the radius of the beam waist, $\rho$ is the volume concentration of the solution, and $l$ is the effective optical path of the laser through the sample:

$$
l=\int_{l_{1}}^{l_{2}}\left(1+\frac{z^{2}}{z_{0}^{2}}\right)^{-1} \mathrm{~d} z, \quad z_{0}=\frac{\pi \omega_{0}^{2}}{\lambda} .
$$

$l_{1}$ and $l_{2}$ are the distances from the front surface and the back surface of the cuvette to the lens, respectively. The number of effective nanosheets is related to the total number of fragments and the incident light intensity $I_{0}$ within the effective length $l$. The proportion of active flakes, $P_{\text {total }}$, is a function of the incident light intensity $I$ and the effective optical path $l^{[20]}$ :

$$
A_{\text {eff }}=A_{\text {total }}(l, \rho) \times P_{\text {total }}(I, l) .
$$

At a fixed concentration, the number of SSPM rings is proportional to the number of fragments $M$, which have already generated electron coherence. The light intensity determines the number of fragments that can generate electron coherence. Still, it is not inversely proportional to $M$, because the change in light intensity changes the number of coherent fragments and the number of SSPM rings simultaneously (at different intensities, producing one ring needs different $M$ ). Therefore, we introduced the proportionality coefficient $\chi$. Finally, it is organized into

$$
M=\frac{N}{I} \chi
$$

Moreover, according to the optical Kerr effect, the nonlinear refractive index $n_{2}$ can be described as ${ }^{[33]}$

$$
n_{2}=\frac{\lambda}{2 n_{0} l} \cdot \frac{N}{I}
$$

where $\lambda$ is the excitation wavelength, and $n_{0}$ is the linear refractive index. As can be seen in Eq. (6), the proportionality coefficient $\chi$ is related to parameters of the sample, such as the nonlinear refractive index.

In the ideal model, the fragments in the solution are evenly distributed from 0 to $\pi / 2$. At different concentrations, the average rotational angle of fragments that generated electron coherence $\varphi$ should be proportional to the effective amount of fragments $M$. Therefore, the following equation can be obtained:

$$
\frac{\varphi}{\pi / 2}=\frac{M}{\rho \pi R^{2} l / \pi r^{2} h},
$$

which finally turns into ${ }^{[2]}$

$$
\begin{gathered}
\varphi=\frac{\left(\varepsilon_{r}-1\right)}{\varepsilon_{r}} \frac{2 t I h}{4 \eta \xi r c} \cdot N \cdot \overline{\sin 2 \theta_{i}}, \\
T=t \cdot N \\
T=\frac{n \pi \chi \varepsilon_{r} \eta \xi r^{3} c}{0.86\left(\varepsilon_{r}-1\right) I^{2} R^{2} l \rho} .
\end{gathered}
$$

As can be seen from Fig. 3(a), the number of SSPM rings keeps increasing constantly as the concentration remains relatively small. As the concentration reaches the saturation value at $0.2 \mathrm{~cm}^{3} / L$ or so, the number of rings no longer increases. In Fig. 3(b), the formation time of diffraction rings decreases with increasing concentration. It is concluded that specific light intensity can make a limited number of $\mathrm{MoS}_{2}$ fragments produce electronic coherence. When the light intensity is saturated, the SSPM rings can no longer change even if the concentration
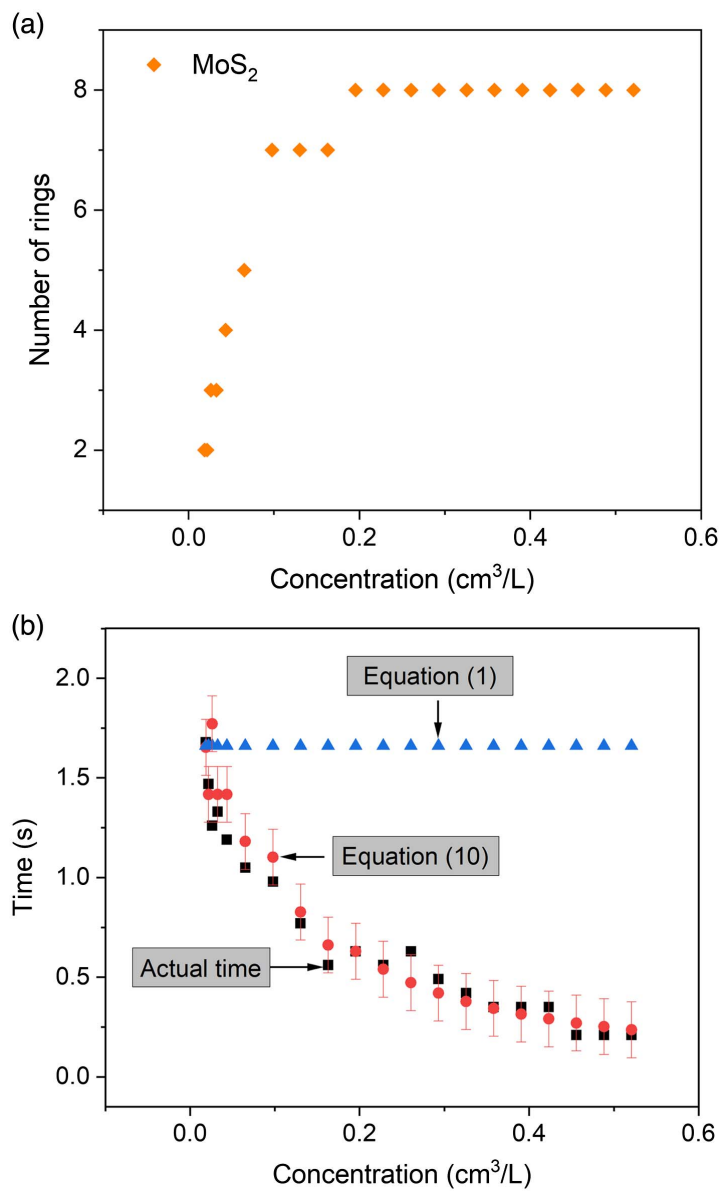

Fig. 3. (a) Number of rings versus different concentrations. (b) The experimental values and two kinds of theoretical values of ring formation time at different concentrations. 
increases. There are three concentrations corresponding to the number of rings of seven because the concentration has a small effect on the number of rings ${ }^{[20]}$.

In Fig. 3(b), the black points represent the experimental values, the red dots represent the theoretical values calculated by Eq. (10), and the blue dots represent the theoretical values calculated by Eq. (1). Through experiments, we found that the formation time will decrease as the concentration increases under the same light intensity. Especially at high concentrations $\left(0.52 \mathrm{~cm}^{3} / \mathrm{L}\right)$, the theoretical result of the original formula $(1.66 \mathrm{~s})$ is about eight times the experimental value $(0.21 \mathrm{~s})$.

It is difficult for the original formula to calculate the formation time of the diffraction rings precisely under different concentrations.

Comparing the theoretical and experimental values, it is found that the theoretical value can also show the trend of the change in theoretical data such as $0.26 \mathrm{~cm}^{3} / \mathrm{L}$ and $0.032 \mathrm{~cm}^{3} / \mathrm{L}$, which indirectly verifies the credibility of the formula presented in this work. The error of the data largely comes from lowconcentration samples because there are fewer 2D fragments. When the sample concentration is low, the proportion of thermal effects increases, and the nonlinear optical characteristics are weakened. Since the thermal effect is not considered in this article, there is a difference between the experimental data and the calculated data.

Equation (10) can be used to accurately fit the relationship between the formation time and the sample concentration at the same light intensity. The formation time of the diffraction rings is related to the sample concentration and closely related to the incident light intensity. Using this formula, the experimental values for the different incident light intensities are compared with the theoretical values under the condition that $\mathrm{MoS}_{2}$ concentration is consistent with the value of $\chi$ in the calculation process. Figure 4 displays the comparison of the theoretically calculated value and experimental value at the concentrations

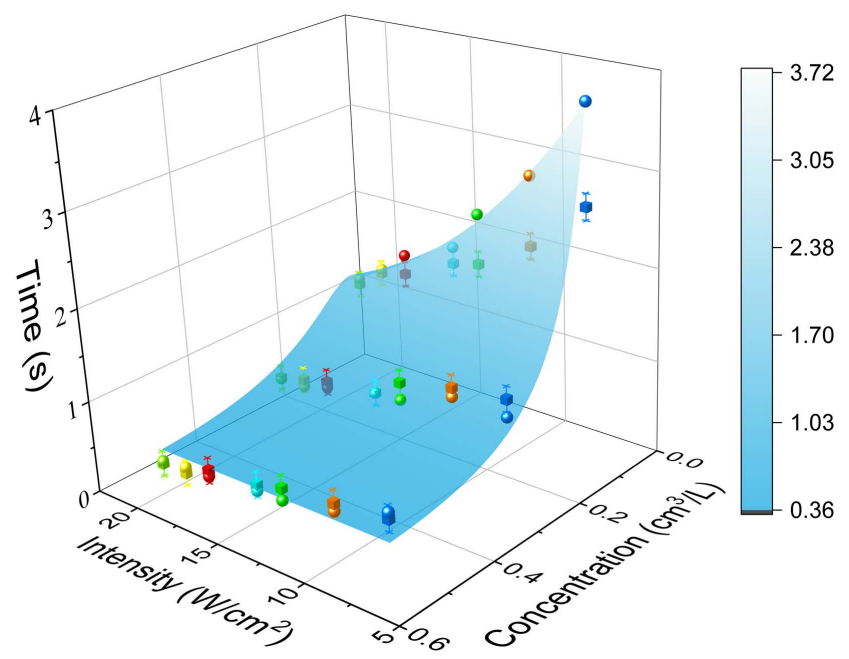

Fig. 4. Time varying with light intensity and concentration. The cubic dots represent the experimental values, and the spherical dots represent the theoretical values. of $0.52 \mathrm{~cm}^{3} / \mathrm{L}, 0.26 \mathrm{~cm}^{3} / \mathrm{L}$, and $0.065 \mathrm{~cm}^{3} / \mathrm{L}$. At high concentration, the difference between the theoretical and experimental values is within the error range $(0-0.14 \mathrm{~s})$. With the decrease of light intensity at low concentration, the gap between the theoretical value and the experimental value becomes larger and larger and is gradually distorted.

\section{Conclusions}

Combining all of the above results, we found that the formation time of the diffraction rings gradually decreases with the increase in light intensity and the increase in the concentration. The surface fitted by a small amount of experimental data can predict the formation time of the diffraction rings under specific concentrations and intensities. In other words, it provides a facile way to find the condition of concentration and light intensity corresponding to the shortest formation time. Due to the limitation of our detector's resolution, we cannot observe a response time less than $0.07 \mathrm{~s}$. There are saturation values for both light intensity and concentration, and hence from Eq. (10), the response time cannot be infinitesimally small. Besides, due to the complexity of the formula, the formation time cannot become infinitely small by only changing the concentration. But, the viscosity coefficient can be infinitely large. In other words, the reorientation of fragments is eliminated by preparing solid films. In this way, the number of rings will not increase over time, and the response time will be significantly reduced. The method we proposed is theoretically feasible, though it can be complicated to get diffraction ring patterns in solid materials.

Using the experimental data of $\mathrm{MoS}_{2}$ at different concentrations, a new unsaturated wind-chime model was successfully established. By comparison, the calculated data is consistent with the experimental data, verifying the feasibility of the formula at different materials and different concentrations. At the same time, the formula is also applicable for calculating the ring formation time of the $2 \mathrm{D}$ layered material at different intensities. Finally, the shortest formation time of diffraction rings can be predicted by limited experimental data. The results obtained herein have advanced significance for photonic devices such as photodiodes.

\section{Acknowledgement}

This work was financially supported by the National Natural Science Foundation of China (Nos. 61875232, 61874141, and 11904239) and the China Postdoctoral Science Foundation (No. 2021M690169).

\section{References}

1. S. D. Durbin, S. M. Arakelian, and Y. R. Shen, "Laser-induced diffraction rings from a nematic-liquid-crystal film," Opt. Lett. 6, 411 (1981).

2. Y. Wu, Q. Wu, F. Sun, C. Cheng, S. Meng, and J. Zhao, "Emergence of electron coherence and two-color all-optical switching in $\mathrm{MoS}_{2}$ based on spatial self-phase modulation," Proc. Natl. Acad. Sci. USA 112, 11800 (2015). 
3. S. Xiao, Y. Zhang, Y. Ma, Y. Wang, Y. He, J. Zhang, Y. Jiang, X. Li, R. Yang, $\mathrm{J}$. He, and Y. Wang, "Observation of spatial self-phase modulation induced via two competing mechanisms," Opt. Lett. 45, 2850 (2020).

4. G. Wang, S. Zhang, F. A. Umran, X. Cheng, N. Dong, D. Coghlan, Y. Cheng, L. Zhang, W. J. Blau, and J. Wang, "Tunable effective nonlinear refractive index of graphene dispersions during the distortion of spatial self-phase modulation," Appl. Phys. Lett. 104, 141909 (2014).

5. Y. Wang, Y. Tang, P. Cheng, X. Zhou, Z. Zhu, Z. Liu, D. Liu, Z. Wang, and J. Bao, "Distinguishing thermal lens effect from electronic third-order nonlinear self-phase modulation in liquid suspensions of 2D nanomaterials," Nanoscale 9, 3547 (2017).

6. S. Xiao, B. Lv, L. Wu, M. Zhu, J. He, and S. Tao, "Dynamic self-diffraction in MoS2 nanoflake solutions," Opt. Express 23, 5875 (2015).

7. G. Wang, S. Zhang, X. Zhang, L. Zhang, Y. Cheng, D. Fox, H. Zhang, J. N. Coleman, W. J. Blau, and J. Wang, "Tunable nonlinear refractive index of two-dimensional $\mathrm{MoS}_{2}, \mathrm{WS}_{2}$, and $\mathrm{MoSe}_{2}$ nanosheet dispersions," Photon. Res. 3, A51 (2015).

8. X. Li, K. Hu, B. Lyu, J. Zhang, Y. Wang, P. Wang, S. Xiao, Y. Gao, and J. He, "Enhanced nonlinear optical response of rectangular $\mathrm{MoS}_{2}$ and $\mathrm{MoS}_{2} / \mathrm{TiO}_{2}$ in dispersion and film," J. Phys. Chem. C 120, 18243 (2016).

9. J. Li, Z. Zhang, J. Yi, L. Miao, J. Huang, J. Zhang, Y. He, B. Huang, C. Zhao, Y. Zou, and S. Wen, "Broadband spatial self-phase modulation and ultrafast response of MXene $\mathrm{Ti}_{3} \mathrm{C}_{2} \mathrm{~T}_{\mathrm{x}}$ ( $\mathrm{T}=\mathrm{O}, \mathrm{OH}$ or $\mathrm{F}$ )," Nanophotonics 9, 2415 (2020).

10. Y. Wang, Y. Wang, K. Chen, K. Qi, T. Xue, H. Zhang, J. He, and S. Xiao, "Niobium-carbide Mxenes with broadband nonlinear optic response and ultrafast carrier dynamics," ACS Nano 14, 10492 (2020).

11. G. Wang, D. Bennett, C. Zhang, C. Ó Coileáin, M. Liang, N. McEvoy, J. J. Wang, J. Wang, K. Wang, and V. Nicolosi, "Two-photon absorption in monolayer Mxenes," Adv. Opt. Mater. 8, 1902021 (2020).

12. L. Wu, W. Huang, Y. Wang, J. Zhao, D. Ma, Y. Xiang, J. Li, J. S. Ponraj, S. C. Dhanabalan, and H. Zhang, "2D tellurium based high-performance all-optical nonlinear photonic devices," Adv. Func. Mater. 29, 1806346 (2019).

13. W. Wang, Y. Wu, Q. Wu, J. Hua, and J. Zhao, "Coherent nonlinear optical response spatial self-phase modulation in $\mathrm{MoSe}_{2}$ nano-sheets," Sci. Rep. 6, 22072 (2016)

14. B. Shi, L. Miao, Q. Wang, J. Du, P. Tang, J. Liu, C. Zhao, and S. Wen, "Broadband ultrafast spatial self-phase modulation for topological insulator $\mathrm{Bi}_{2} \mathrm{Te}_{3}$ dispersions," Appl. Phys. Lett. 107, 151101 (2015).

15. X. Li, R. Liu, H. Xie, Y. Zhang, B. Lyu, P. Wang, J. Wang, Q. Fan, Y. Ma, S. Tao, S. Xiao, X. Yu, Y. Gao, and J. He, "Tri-phase all-optical switching and broadband nonlinear optical response in $\mathrm{Bi}_{2} \mathrm{Se}_{3}$ nanosheets," Opt. Express 25, 18346 (2017).

16. J. Zhang, X. Yu, W. Han, B. Lv, X. Li, S. Xiao, Y. Gao, and J. He, "Broadband spatial self-phase modulation of black phosphorous," Opt. Lett. 41, 1704 (2016).

17. J. Li, L. Du, J. Huang, Y. He, J. Yi, L. Miao, C. Zhao, and S. Wen, "Passive photonic diodes based on natural van der Waals heterostructures," Nanophotonics 10, 927 (2021).

18. L. Hu, F. Sun, H. Zhao, and J. Zhao, "Nonlinear optical response spatial selfhase modulation in $\mathrm{MoTe}_{2}$ : correlations between $\chi^{(3)}$ and mobility or effective mass," Opt. Lett. 44, 5214 (2019).

19. Y. Wang, G. Huang, H. Mu, S. Lin, J. Chen, S. Xiao, Q. Bao, and J. He, "Ultrafast recovery time and broadband saturable absorption properties of black phosphorus suspension," Appl. Phys. Lett. 107, 091905 (2015).
20. S. Xiao, Y. Ma, Y. He, Y. Wang, H. Xin, Q. Fan, J. Zhang, X. Li, Y. Zhang, $\mathrm{J} . \mathrm{He}$, and $\mathrm{Y}$. Wang, "Revealing the intrinsic nonlinear optical response of a single $\mathrm{MoS}_{2}$ nanosheet in a suspension based on spatial self-phase modulation," Photon. Res. 8, 1725 (2020).

21. Y. Shan, J. Tang, L. Wu, S. Lu, X. Dai, and Y. Xiang, "Spatial self-phase modulation and all-optical switching of graphene oxide dispersions," J. Alloy. Compd. 771, 900 (2019).

22. Y. Jia, Z. Li, M. Saeed, J. Tang, H. Cai, and Y. Xiang, "Kerr nonlinearity in germanium selenide nanoflakes measured by $Z$-scan and spatial self-phase modulation techniques and its applications in all-optical information conversion," Opt. Express 27, 20857 (2019).

23. Z. Du, H. Wu, T. Zhang, Z. Xie, Y. Lü, X. Lü, J. Xu, G. Zhao, and S Zhu, "Ultra-broadband enhanced nonlinear saturable absorption for $\mathrm{Mo}_{0.53} \mathrm{~W}_{0.47} \mathrm{Te}_{2}$ nanosheets," Chin. Opt. Lett. 18, 021902 (2020).

24. L. Gong, X. Zhang, Z. Zhu, G. Rui, J. He, Y. Cui, and B. Gu, "Femtosecond laser trapping dynamics of two-photon absorbing hollow-core nanoparticles," Chin. Opt. Lett. 18, 081901 (2020).

25. S. Hua, K. Du, H. Wang, W. Zhang, T. Mei, and E. Dogheche, "Affirming nonlinear optical coefficient constancy from Z-scan measurement," Chin. Opt. Lett. 18, 071903 (2020).

26. Y. Wang, H. Mu, X. Li, J. Yuan, J. Chen, S. Xiao, Q. Bao, Y. Gao, and J. He, "Observation of large nonlinear responses in a graphene- $\mathrm{Bi}_{2} \mathrm{Te}_{3}$ heterostructure at a telecommunication wavelength," Appl. Phys. Lett. 108, 221901 (2016).

27. L. Wu, X. Yuan, D. Ma, Y. Zhang, W. Huang, Y. Ge, Y. Song, Y. Xiang, J. Li, and $\mathrm{H}$. Zhang, "Recent advances of spatial self-phase modulation in $2 \mathrm{D}$ materials and passive photonic device applications," Small 16, 2002252 (2020).

28. X.-J. Zhang, Z.-H. Yuan, R.-X. Yang, Y.-L. He, Y.-L. Qin, S. Xiao, and J. He, "A review on spatial self-phase modulation of two-dimensional materials," J. Central South University 26, 2295 (2019).

29. Y. Shan, L. Wu, Y. Liao, J. Tang, X. Dai, and Y. Xiang, "A promising nonlinear optical material and its applications for all-optical switching and information converters based on the spatial self-phase modulation (SSPM) effect of $\mathrm{TaSe}_{2}$ nanosheets," J. Mater. Chem. C 7, 3811 (2019).

30. L. Wu, Y. Dong, J. Zhao, D. Ma, W. Huang, Y. Zhang, Y. Wang, X. Jiang, $\mathrm{Y}$. Xiang, and J. Li, "Kerr nonlinearity in 2D graphdiyne for passive photonic diodes," Adv. Mater. 31, 1807981 (2019).

31. L. Wu, Y. Zhang, X. Yuan, F. Zhang, W. Huang, D. Ma, J. Zhao, Y. Wang, Y. Ge, and H. Huang, "1D@0D hybrid dimensional heterojunctionbased photonics logical gate and isolator," Appl. Mater. Today 19, 100589 (2020).

32. J. N. Coleman, M. Lotya, A. O'Neill, S. D. Bergin, P. J. King, U. Khan, K. Young, A. Gaucher, S. De, R. J. Smith, I. V. Shvets, S. K. Arora, G. Stanton, H.-Y. Kim, K. Lee, G. T. Kim, G. S. Duesberg, T. Hallam, J. J. Boland, J. J. Wang, J. F. Donegan, J. C. Grunlan, G. Moriarty, A. Shmeliov, R. J. Nicholls, J. M. Perkins, E. M. Grieveson, K. Theuwissen, D. W. McComb, P. D. Nellist, and V. Nicolosi, "Twodimensional nanosheets produced by liquid exfoliation of layered materials," Science 331, 568 (2011)

33. R. Wu, Y. Zhang, S. Yan, F. Bian, W. Wang, X. Bai, X. Lu, J. Zhao, and E. Wang, "Purely coherent nonlinear optical response in solution dispersions of graphene sheets," Nano Lett. 11, 5159 (2011). 